\title{
Superpositions of Chiral Molecules
}

\author{
Matter-wave diffraction can put chiral molecules into superpositions of \\ left- and right-handed forms, enabling new studies of how the two states \\ interact with their environment.
}

By Christopher Crockett

M any complex molecules come in two configurations that look like mirror images of each other. The leftand right-handed versions of these so-called chiral molecules can behave very differently from each other in biological settings. Now, researchers show how to prepare a beam of chiral molecules in a quantum superposition of leftand right-handed variations that could enable precision studies of these differences [1].

The proposed experiment would leverage a unique quantum property of chiral molecules: They oscillate periodically between the two forms. When not fully left- or right-handed, they are in a quantum superposition of both states. Each molecule in a beam, however, is in a different stage of these oscillations. If it were possible to coordinate this cycling so that many molecules switched configurations in sync, one would have a beam of chiral molecules in well-defined states.

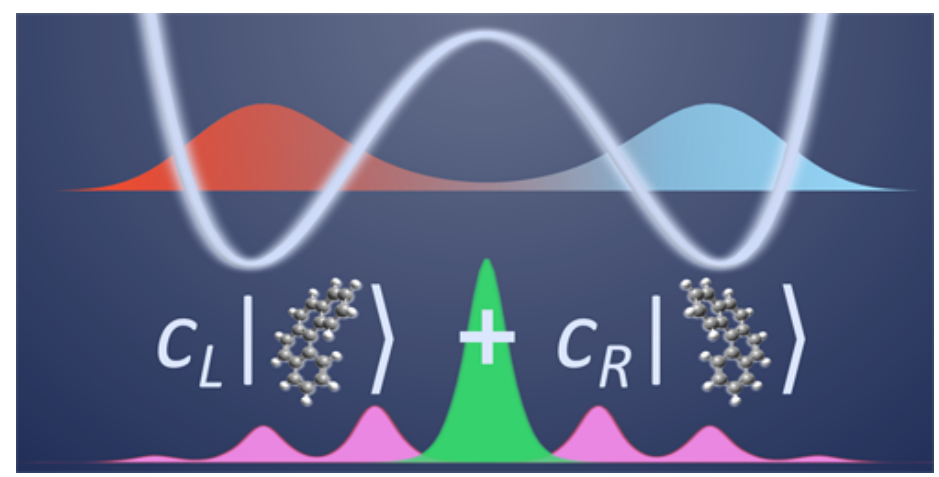

Credit: Daqing Wang/University of Kassel
Benjamin Stickler of the University of Duisburg-Essen in Germany and colleagues propose to achieve this synchronization by aiming a beam of chiral molecules at a specially tailored laser-based diffraction grating. Molecules reaching the grating in their left-handed forms would go to one diffraction order and those in their right-handed forms to another. Spatial filtering of these orders would select molecules of a certain state: The filtered molecules would continue to flip between forms, but they would now do so in sync. This filtered beam could then be used to sense chiral-dependent interactions, which would introduce phase shifts between the left- and right-handed states.

The team suggests that derivatives of the helix-shaped molecule [4]-helicene will be ideal for this work: The oscillation times are suitable for exploiting chiral quantum superpositions, and the strong optical response means that a moderate-intensity laser can separate the configurations.

Christopher Crockett is a freelance writer based in Arlington, Virginia.

\section{REFERENCES}

1. B. A. Stickler et al., "Enantiomer superpositions from matter-wave interference of chiral molecules," Phys. Rev. X 11, 031056 (2021). 